\title{
Editorial: \\ Iran-Cohort Study of COVID-19 Immunology and Immunopathology
}

Tooba Ghazanfari ${ }^{*}$

1. Immunoregulation Research Center, Shahed University, Tehran, Iran.

Citation Ghazanfari T. Iran-Cohort Study of COVID-19 Immunology and Immunopathology. Immunoregulation. 2020; 3(1):1-2. http://dx.doi.org/10.32598/Immunoregulation.3.1.1

http://dx.doi.org/10.32598/Immunoregulation.3.1.1

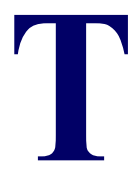

he viral disease of severe acute respiratory syndrome coronavirus 2 (SARSCoV-2) or COVID-19 was identified in early December 2019 in Wuhan, China, and quickly spread worldwide, and became a pandemic after 3 months. COVID-19 presents a wide range of clinical manifestations from asymptomatic infection to severe pneumonia accompanied by multi-organ failure that can lead to death. The rapid spread of COVID-19, on the one hand, and its clinical complications, on the other hand, has posed a serious threat to human health.

Besides the signs and symptoms of the severe acute respiratory syndrome (SARS) secondary to other beta coronaviruses, including fever, myalgia, cough, and sore throat, the COVID-19, which is phylogenetically related to SARS-induced coronaviruses such as SARS-CoV-2, shares same entry receptor called the angiotensin-converting enzyme receptor, yet with more affinity. Considering the immunomodulatory effect of this enzyme, SARS-CoV-2 by attachment to this receptor causes immune dysregulation, disruption of immune cells ratio, in particular lymphopenia, and derangement of cytokine networks such as the exponential increase in interleukin 6 (IL-6), out of the control excretion of IL- 6 together with the disruption of its ratio to other inflammatory cytokines. The balance between the protective immune response and exacerbation of the inflammatory components determines the severity of the disease.

The critical point in COVID-19 is that people at risk of this disease are men, elderly, with comorbidities like hypertension, cardiovascular disease, diabetes, chronic obstructive pulmonary disease, and chronic kidney disease. Studies have shown that patients with these risk factors are severely affected by the SARS-CoV-2, with subsequent severe failure in organs such as lungs, heart, liver, and kidney, and an increase in the odds of mortality.

In all of those aforementioned comorbidities, there is a disorder in the immune responses, and it looks like that these disorders provide a strong foothold for the SARSCoV-2 in the human body. On the other hand, no confirmatory evidence has so far been found in patients with other immune dysregulations such as asthma, autoimmune rheumatic disease, and immunodeficiency.

Therefore, to adopt useful strategies for the prevention, treatment, and improvement of the COVID-19, and providing the vaccination of eligible individuals, there is an urgent need to identify and evaluate the exact immunology and immunopathology status in patients with $\mathrm{CO}$ VID-19 in different populations. Also, it is necessary to identify the immunopathology and immunophenotyping of damaged tissues, as well as the determination of the

* Corresponding Author:

Tooba Ghazanfari, PhD.

Address: Immunoregulation Research Center, Shahed University, Tehran, Iran.

Phone: +98 (21) 66418216

E-mail:tghazanfari@yahoo.com 
presence, type, level, and duration of the specific protective immune responses.

In response to this concern, a large multicenter cohort study entitled "Iran-Cohort Study of COVID-19 Immunology and Immunopathology" was started to evaluate a comprehensive set of clinical and laboratory biomarkers and specific immune responses over the time. One of the articles of this study regarding the impact of IL-6 on disease severity has been published in this issue of the journal. 